\title{
Diferencias significativas en el uso de redes sociales entre universitarios españoles y colombianos
}

\begin{abstract}
Alba Soraya Aguilar-Jiménez, Marianela Luzardo-Briceño ${ }^{\text {, }}$, Ludym JaimesCarrillo $^{c}$, Luis Matosas-López ${ }^{\mathrm{d}}$

${ }^{a}$ Grupo de Investigación GeeTIC, Facultad de Ingeniería Industrial, Universidad Pontificia Bolivariana Seccional Bucaramanga Colombia. Email: alba.aguilar@upb.edu.co. ${ }^{\mathrm{b}}$ Grupo de Investigación GeeTIC, Facultad de Ingeniería Industrial, Universidad Pontificia Bolivariana

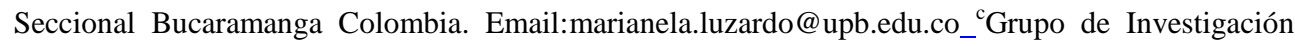
GeeTIC, Facultad de Ingeniería Industrial, Universidad Pontificia Bolivariana Seccional Bucaramanga Colombia. Email: ludym.jaimes@upb.edu.co_d Departamento de Economía Financiera, Contabilidad e Idioma Moderno, Universidad Rey Juan Carlos, Madrid, España. Email: luis.matosas@urjc.es
\end{abstract}

\section{Resumen}

La fuerte influencia de las redes sociales en todos los ámbitos de la sociedad moderna ha convertido a estas plataformas en protagonistas de un fenómeno de dimensiones globales que traspasa fronteras, para afianzarse a ambos lados del Atlántico. El presente trabajo examina las posibles diferencias en el uso que, hoy por hoy, hacen los universitarios españoles y colombianos de estas plataformas.

En la investigación toman parte 425 estudiantes: 198 de Administración y Dirección de Empresas de la Universidad Rey Juan Carlos en España y 227 de Ingeniería Industrial de la Universidad Pontificia Bolivariana en Colombia. En ambas instituciones, los participantes completan un cuestionario, elaborado ad hoc por los investigadores a partir de una revisión previa de la literatura. El cuestionario recoge, por un lado, datos relativos a la frecuencia de acceso a las redes sociales de referencia (Twitter, Facebook e Instagram) y, por otro, información tocante a la importancia que el estudiante otorga a determinados usos de la plataforma. Los datos recabados son sometidos a análisis descriptivo e inferencial paramétrico para muestras independientes empleando la prueba t-Student.

Los resultados obtenidos revelan la existencia de diferencias significativas en diversos puntos relativos a la utilización que los estudiantes de ambos países hacen de las redes sociales objeto de análisis. Estas diferencias se observan, por ejemplo, en la frecuencia de acceso a las redes Facebook e Instagram; plataformas en las que los estudiantes españoles acceden con 
mayor asiduidad que los colombianos. Asimismo, el estudio muestra diferencias significativas en ambos países en lo que atañe a la importancia otorgada a la funcionalidad "me gusta", donde el mayor uso promedio corresponde a los estudiantes de la URJC, y en cuanto a la participación en debates a través de estas plataformas el comportamiento es inverso.

Palabras claves: Redes sociales, estudiantes universitarios

\section{Introducción}

Internet y el avance tecnológico han venido revolucionado los diferentes sectores económicos. El sector educativo no es la excepción. Así las universidades han sido unas de las grandes beneficiadas por la tecnología en el ámbito científico y pedagógico (GuzmánDuque, Alba Patricia; Luzardo Briceño, Marianela; Aguilar-Jiménez, 2013).

La evolución de la web y las facilidades de interacción que trajo consigo la web 2.0 son un aporte a las ciencias. Por un lado, porque facilitan la participación de los usuarios y, por otro, porque permiten la interacción entre ellos (Musser \& O'reilly, 2007). En este sentido, cabe mencionar que el trabajo colaborativo en ambientes virtuales incluye un variado rango de temas de estudio y de metodologías que fomentan la autonomía en el aprendizaje. Los usuarios de la web y en concreto, de plataformas tales como blogs, wikis, redes sociales, chats, etc.- acceden diariamente para compartir experiencias y gustos en común (Christodoulou \& Styliaras, 2008) comunicándose e interactuando.

En este sentido, la integración de las Tecnologías de Información y Comunicación (TIC) en la práctica docente a nivel universitario es un tema que ha venido siendo estudiado desde diversas perspectivas, no solo a nivel tecnológico sino también didáctico en las universidades (Luzardo Briceño, Sandia Saldivia, Aguilar-Jiménez, Macías Martínez, \& Herrera Díaz, 2017; Villarraga Plaza, Aguilar-Jiménez, Luzardo Briceño, Sandia Saldivia, \& Del Alba Catherin, 2017), mostrando resultados que invitan a los docentes a considerar las TIC en su práctica pedagógica.

Si bien las TIC tienen un potencial importante para los procesos académicos, estudios anteriores han identificado que los jóvenes prefieren el uso de las TIC en actividades de ocio (Sanz Arazuri, Alonso Ruiz, Saénz de Jubera Ocón, Ponce de León Elizondo, \& Valdemoros San Emeterio, 2018). Por tanto, la tecnología, en la actualidad, aún tiene mucho por aportar tanto a docentes como a estudiantes en su incorporación de nuevas didácticas en los procesos educativos. De hecho, hay estudios que revelan que los estudiantes que tienen acceso y usan las TIC en casa muestran mejor rendimiento académico que quienes no lo hacen (Alderete, Di Meglio, \& Formichella, 2017). 
En este sentido, el presente documento pretende examinar las posibles diferencias en el uso que, hoy por hoy, hacen los universitarios españoles y colombianos de herramientas TIC, en particular de los medios sociales.

\section{Marco de referencia}

Si bien el uso de las TIC como apoyo a actividades académicas ha venido creciendo, es probable que aún no estén impactando significativamente en la educación universitaria. Es claro también que en este proceso no solo influye el interés pedagógico (Luzardo Briceño et al., 2017; Menéndez, Díaz, Sánchez, \& Linares, 2014; Sancho Gil, 2011) y la dinámica tecnológica (Velandia, Ríos, \& de León, 2010; Villarraga Plaza et al., 2017), sino también el compromiso organizacional (Luzardo-Briceño, Aguilar-Jiménez, Sandia-Saldivia, \& Acosta-Santiago, 2017), cuyo cambio cultural es necesario para que las TIC impacten de manera significativa en la formación (Sorroza Rojas, Nancy Azucena; Jinez Sorroza, Jean Pool; Rodríguez Villacis, Jesús Eliecer; Caraguay Ambuludi, 2018).

Las herramientas de la web 2.0 o de los medios sociales -blogs, wikis, foros, Flickr, Facebook, Delicious entre otras- son funcionales para las universidades (Siemens \& Tittenberger, 2009). Asimismo estudios anteriores sugieren que el uso de plataformas sociales ayuda a obtener mejor rendimiento (por ejemplo, para la comunicación con familia y amigos) aumentando las probabilidades de adopción en el futuro (Calderón, López, \& Peña, 2017).

Según estudios anteriores, los estudiantes españoles valoran positivamente las herramientas web 2.0, blog y/o edublog, WebQuest y wiki, para promover las estrategias de los entornos educativos inclusivos, así como el uso de redes sociales con fines académicos (Requena \& Berea, 2017). Por su parte, los estudiantes universitarios de en Colombia no parecen apropiase de las posibilidades productivas de estas plataformas más allá de los usos básicos o recreativos (Berrío Zapata \& Rojas Hernández, 2014).

\section{Metodología}

Se realizó una investigación mixta, no experimental, de corte transversal con un alcance exploratorio descriptivo y correlacional.

La muestra estuvo conformada por 425 estudiantes de los cuales 198 pertenecen a los diferentes cursos de Administración y Dirección de Empresas de la Universidad Rey Juan 
Carlos (URJC) en España y 227 forman parte de diferentes cursos de Ingeniería Industrial de la Universidad Pontificia Bolivariana (UPB) en Colombia.

La edad promedio de los estudiantes de la URJC $19.58(D T=2.67)$, con una distribución género de $32.32 \%$ (femenino), 67.68\% (masculino), mientras que en la UPB se situó en $20.77(D T=2.37)$, siendo la distribución de género de $56.39 \%$ (femenino), $43.61 \%$ (masculino).

Los datos recabados para la investigación se obtuvieron empleando un cuestionario de construcción propia, elaborado por los investigadores. El instrumento de medición se sometió a la evaluación de un equipo de jueces expertos en el ámbito de la educación superior, compuesto por ocho profesores: cuatro de la UPB y cuatro de la URJC. El cuestionario final consta de dieciocho ítems que miden aspectos relacionados con el uso de redes sociales en universitarios.

Para la comparación en la puntuación obtenida en el uso de las distintas redes sociales entre los estudiantes de ambas universidades se utilizó la prueba $t$ de Student para muestras independientes del software IBM-SPSS V 24.

\section{Resultados}

La tabla 1 Estadísticos descriptivos uso Redes Sociales presenta los estadísticos descriptivos del uso de las redes sociales consideradas en el estudio.

Los resultados revelan como, en la mayoría de los casos, las puntuaciones promedio obtenidas en la muestra de universitarios colombianos superan a las recogidas en la muestra de estudiantes españoles. 
Tabla 1. Estadísticos descriptivos uso Redes Sociales

\begin{tabular}{|c|c|c|c|}
\hline Ítem & Universidad & Media & $D T$ \\
\hline \multirow{2}{*}{ Frecuencia de uso de Twitter } & URJC & 3.04 & 1.826 \\
\hline & UPB & 2.78 & 2.003 \\
\hline \multirow{2}{*}{ Frecuencia de uso de Facebook } & URJC & 3.71 & 2.021 \\
\hline & UPB & 5.15 & 1.821 \\
\hline \multirow{2}{*}{ Frecuencia de uso de Instagram } & URJC & 4.30 & 1.290 \\
\hline & UPB & 5.48 & 1.906 \\
\hline \multirow{2}{*}{ Empleo de Menciones } & URJC & 2.80 & .945 \\
\hline & UPB & 2.71 & .947 \\
\hline \multirow{2}{*}{ Empleo de Hashtags } & URJC & 2.08 & 1.029 \\
\hline & UPB & 1.93 & .943 \\
\hline \multirow{2}{*}{ Empleo de Likes } & URJC & 3.73 & 1.050 \\
\hline & UPB & 3.42 & 1.054 \\
\hline \multirow{2}{*}{$\begin{array}{l}\text { Relevancia dada a seguir } \\
\text { amigos }\end{array}$} & URJC & 3.80 & 1.069 \\
\hline & UPB & 3.62 & 1.080 \\
\hline \multirow{2}{*}{$\begin{array}{l}\text { Relevancia dada a seguir } \\
\text { personajes públicos }\end{array}$} & URJC & 2.42 & 1.048 \\
\hline & UPB & 2.42 & 1.046 \\
\hline \multirow{2}{*}{$\begin{array}{l}\text { Relevancia dada a menciones a } \\
\text { amigos }\end{array}$} & URJC & 3.30 & .992 \\
\hline & UPB & 3.25 & 1.097 \\
\hline \multirow{2}{*}{$\begin{array}{l}\text { Relevancia dada a menciones a } \\
\text { personajes públicos }\end{array}$} & URJC & 2.12 & 1.102 \\
\hline & UPB & 2.21 & 1.042 \\
\hline \multirow{2}{*}{ Relevancia dada a ver videos } & URJC & 3.31 & 1.057 \\
\hline & UPB & 3.59 & 1.087 \\
\hline \multirow{2}{*}{$\begin{array}{l}\text { Relevancia dada a publicar } \\
\text { videos }\end{array}$} & URJC & 2.30 & 1.139 \\
\hline & UPB & 2.37 & 1.172 \\
\hline \multirow{2}{*}{$\begin{array}{l}\text { Relevancia dada a la búsqueda } \\
\text { de información }\end{array}$} & URJC & 3.72 & 1.075 \\
\hline & UPB & 3.89 & 1.040 \\
\hline \multirow{2}{*}{ Relevancia dada a ver fotos } & URJC & 3.62 & 1.019 \\
\hline & UPB & 3.83 & 1.025 \\
\hline \multirow{2}{*}{ Relevancia dada a publicar fotos } & URJC & 3.06 & 1.214 \\
\hline & UPB V & 3.11 & 1.118 \\
\hline \multirow{2}{*}{$\begin{array}{l}\text { Relevancia dada a publicar } \\
\text { reflexiones personales }\end{array}$} & URJC & 2.22 & 1.161 \\
\hline & UPB & 2.23 & 1.146 \\
\hline \multirow{2}{*}{$\begin{array}{l}\text { Relevancia dada al acceso a } \\
\text { promoaciones }\end{array}$} & URJC & 2.21 & 1.147 \\
\hline & UPB & 2.57 & 1.262 \\
\hline \multirow{2}{*}{$\begin{array}{l}\text { Relevancia dada a la } \\
\text { participación en debates }\end{array}$} & URJC & 1.99 & 1.099 \\
\hline & UPB & 2.51 & 1.191 \\
\hline
\end{tabular}

Fuente:Elaboración propia 
Los resultados muestran que exite una diferencia significativa en el uso promedio de las redes Facebook e Instagram. Asimismo, como se observa en la tabla 2 tambien diferencias significativas en el uso de likes y de la importancia otorgada al medio como instrumento de participación en debates.

Tabla.2 Comparación promedio de uso de redes sociales entre la URJC y UPB

\begin{tabular}{|c|c|c|}
\hline Red social & $t$ & $p$-valor \\
\hline Frecuencia de uso de Twitter & 1.050 & .295 \\
\hline Frecuencia de uso de Facebook & -6.441 & $.000 * * *$ \\
\hline Frecuencia de uso de Instagram & -5.204 & $.000 * * *$ \\
\hline Empleo de Menciones & -.318 & .751 \\
\hline Empleo de Hashtags & 1.048 & .296 \\
\hline Empleo de Likes & 2.191 & $.030 * *$ \\
\hline Relevancia dada a seguir amigos & .660 & .510 \\
\hline Relevancia dada a seguir personajes públicos & -.862 & .390 \\
\hline Relevancia dada a menciones a amigos & .460 & .646 \\
\hline $\begin{array}{l}\text { Relevancia dada a menciones a personajes } \\
\text { públicos }\end{array}$ & -1.714 & $.088 *$ \\
\hline Relevancia dada a ver videos & -.069 & .945 \\
\hline Relevancia dada a publicar videos & -.781 & .436 \\
\hline Relevancia dada a la búsqueda de información & -1.070 & .286 \\
\hline Relevancia dada a ver fotos & -.461 & .645 \\
\hline Relevancia dada a publicar fotos & -.472 & 637 \\
\hline $\begin{array}{l}\text { Relevancia dada a publicar reflexiones } \\
\text { personales }\end{array}$ & -.445 & .656 \\
\hline Relevancia dada al acceso a promoaciones & -.804 & .422 \\
\hline Relevancia dada a la participación en debates & -2.667 & $0.008 * * *$ \\
\hline
\end{tabular}

*** Significativa al $1 \%$

** Significativa al 5\%

*Significativa al $10 \%$

Fuente:Elaboración propia 


\section{Conclusiones}

El uso de las redes sociales esta cada vez más aceptado por los estudiantes universitarios como un mecanismo de comunicación para llegar a sus grupos de interés, en especial aquellas que favorecen la interacción y participación entre ellos.

El análisis estadístico, desarrollado por los autores, muestra la existencia de diferencias significativas en el uso promedio que hacen los estudiantes españoles de la URJC, en comparación con el uso realizado por los estudiantes colombianos de la UPB. Estas diferencias se observan principalmente en la frecuencia de acceso a las redes Facebook e Instagram; plataformas en las que los estudiantes españoles acceden con mayor asiduidad que los colombianos.

Asimismo, el estudio muestra diferencias significativas en ambos países en lo que atañe a la importancia otorgada a la funcionalidad "me gusta" y la participación en debates a través de estas plataformas. En el primer caso, son los estudiantes de la URJC los que dan mayor relevancia a esta utilidad de reconocimiento del contenido. En el segundo, son los universitarios de la UPD los que muestran mas interés por el componente de discusión y debate en las redes sociales objeto de observación.

\section{Referencias}

Alderete, M. V., Di Meglio, G., \& Formichella, M. M. (2017). Acceso a las TIC y rendimiento educativo: ¿ una relación potenciada por su uso? Un análisis para España ICT access and educational performance: $\measuredangle$ a relationship enhanced by ICT use? An analysis for Spain. Revista de Educación, 377, 54-81.

Berrío Zapata, C., \& Rojas Hernández, H. (2014). La brecha digital universitaria: La apropiación de las TIC en estudiantes de educación superior en Bogotá (Colombia).

Calderón, C. A., López, M., \& Peña, J. (2017). El efecto condicional indirecto de la expectativa de rendimiento en el uso de Facebook, Google+, Instagram y Twitter por jóvenes. Revista Latina de Comunicación Social, (72), 590-607.

Christodoulou, S. P., \& Styliaras, G. D. (2008). Digital art 2.0: art meets web 2.0 trend. In Proceedings of the 3rd international conference on Digital Interactive Media in Entertainment and Arts (pp. 158-165). ACM.

Guzmán-Duque, Alba Patricia; Luzardo Briceño, Marianela; Aguilar-Jiménez, A. S. (2013). Un acercamiento a los medios sociales como herramienta de comunicación de los profesores. Caso Universidad Pontificia Bolivariana - Bucaramanga. In GSTIC. Manizalez, Colombia.

Luzardo-Briceño, M., Aguilar-Jiménez, A. S., Sandia-Saldivia, B. E., \& Acosta-Santiago, B. L. (2017). Factores que influyen en la adopción de las TIC por parte de las 
universidades desde la dimensión organizacional. Revista Academia, 16(38).

Luzardo Briceño, M., Sandia Saldivia, B. E., Aguilar-Jiménez, A. S., Macías Martínez, M., \& Herrera Díaz, J. (2017). Factores que influyen en la adopción de las Tecnologías de Información y Comunicación por parte de las universidades. Dimensión EnseñanzaAprendizaje. Educere, 21(68), 143-153.

Menéndez, C. R., Díaz, P. B., Sánchez, Y. S., \& Linares, Y. P. (2014). La enseñanza de estrategias de aprendizaje, una perspectiva pedagógica para las transformaciones en la Educación Superior en Cuba. Pedagogía Universitaria, 19(2).

Musser, J., \& O’reilly, T. (2007). Web 2.0: Principles and best practices. O'Reilly Media,.

Requena, B. E. S., \& Berea, G. A. M. (2017). Valoraciones de los estudiantes mexicanos y españoles sobre el uso de las TIC como recurso para trabajar la educación inclusiva. Bordón. Revista de Pedagogía, 69(3), 89-106.

Sancho Gil, J. M. (2011). Las tic en la universidad desde las experiencias de vida profesional de docentes e investigadores *, (2010), 355-368.

Sanz Arazuri, E., Alonso Ruiz, R. A., Saénz de Jubera Ocón, M., Ponce de León Elizondo, A., \& Valdemoros San Emeterio, M. Á. (2018). Ocio, Redes Sociales Y Estudiantes Españoles. Educación XX1, 21(2). https://doi.org/10.5944/educxx1.19538

Siemens, G., \& Tittenberger, P. (2009). Handbook of emerging technologies for learning. University of Manitoba Winnipeg.

Sorroza Rojas, Nancy Azucena; Jinez Sorroza, Jean Pool; Rodríguez Villacis, Jesús Eliecer; Caraguay Ambuludi, W. A. S. M. V. (2018). Las Tic y la resistencia al cambio en la Educación Superior. Recimundo.Com, 2(2), 477-495. Retrieved from http://www.recimundo.com/index.php/es/article/view/241

Velandia, S. Á. T., Ríos, C. B., \& de León, O. G. P. (2010). Infraestructura tecnológica y apropiación de las TIC en la Universidad Autónoma del Estado de Morelos. Perfiles Educativos, 32(127), 105-127.

Villarraga Plaza, A., Aguilar-Jiménez, A. S., Luzardo Briceño, M., Sandia Saldivia, B., \& Del Alba Catherin. (2017). Factors influencing the adoption of ICT by universities from the technological infrastructure dimension. Journal of Science Education, 18(2), 75-80. 